\title{
Modelagem dos Fatores de Transmissão e DLG para um
} TrueBeam STX Modeling of transmission and DLG factors for a TrueBeam STX

\author{
Laís B.Silva1, Guilherme A. Pavan¹, André V. Camargo¹, Letícia M. Santoni, \\ Diego C. S. A. Silva
}

${ }^{1}$ Hospital de Câncer de Barretos, Barretos, Brasil

\begin{abstract}
Resumo
Para Multi Leaf Collimator (MLC) de ponta arredondada, o sistema de planejamento Eclipse (Varian) exige a medida do Fator de transmissão (FT) e Dosimetric Leaf Gap (DLG) para Intensity Modulated Radiation Therapy (IMRT) e Volumetric Modulated Arc Therapy (VMAT). O uso desses fatores obtidos pela metodologia proposta pela Varian, pode não garantir a melhor concordância em controles da qualidade (CQ) de pacientes, enquanto a modelagem dos mesmos possibilita obter um valor "ótimo" reduzindo as diferenças entre doses medida/calculada. O objetivo desse trabalho foi medir o FT e DLG de um acelerador TrueBeam (TB) com diferentes setups e detectores, modelar esses parâmetros e validar os resultados aplicando a metodologia da AAPM-TG119 utilizando o sistema Portal Dosimetry (PD). Para obtenção do FT e DLG (6MV, 6FFF, 10MV e 10FFF), utilizaram-se planos DICOM fornecidos pelo fabricante e câmara de ionização Farmer e CC13 em diferentes setups. Para a modelagem, inicialmente utilizou-se a dose medida com a câmara Farmer em planejamentos de VMAT de próstata e cabeça e pescoço. A partir dos dados obtidos, interativamente os valores do FT e DLG foram alterados buscando maior índice de aceite (>95\%) no critério gama $1 \% 1 \mathrm{~mm}$ usando o PD. Finalmente, todos os planejamentos do TG119 com IMRT e VMAT foram medidos usando PD. Os valores finais medidos/modelados foram: $\quad 6 X[(F T=1.1 \% / 1.0 \%)(D L G=0.044 \mathrm{~cm} / 0,12 \mathrm{~cm})], \quad 10 X[(F T=1.3 \% / 1,3 \%)$ $(\mathrm{DLG}=0.055 \mathrm{~cm} / 0,13 \mathrm{~cm})], \quad 6 \mathrm{FFF}[(\mathrm{FT}=0.93 \% / 0,4 \%)(\mathrm{DLG}=0.036 \mathrm{~cm} / 0,05 \mathrm{~cm})], \quad 10 \mathrm{FFF}[(\mathrm{FT}=1,14 \% / 1,05 \%)$ $(\mathrm{DLG}=0.048 \mathrm{~cm} / 0,05 \mathrm{~cm})]$. A média das porcentagens de aceite para o critério gamma $1 \% 1 \mathrm{~mm}$ dos planejamentos do TG119 com VMAT/IMRT após a modelagem foram 6X: 96\%/76\%; 10X: 98\%/95\%; 6FFF: $97 \% / 87 \%$; 10FFF: 97\%/82\%. A modelagem do FT e DLG é útil para melhorar a precisão dos controles da qualidade dos pacientes, porém, o detector de escolha e a técnica usada (VMAT ou IMRT) são determinantes e levam a diferentes resultados. Um equilíbrio deve ser encontrado de acordo com a rotina do departamento.
\end{abstract}

Palavras-chave: radioterapia; VMAT; fator de transmissão; Dosimetric Leaf Gap; modelagem.

\begin{abstract}
For the rounded leaf-end Multi Leaf Collimator (MLC), the Eclipse planning system (Varian) requires the measurement of the Transmission Factor (FT) and Dosimetric Leaf Gap (DLG) for Intensity Modulated Radiation Therapy (IMRT) and Volumetric Modulated Arc Therapy (VMAT). The use of these factors obtained by the methodology proposed by Varian may not guarantee the best agreement in quality assurence (QA) of patients, while the modeling of these factors makes it possible to obtain an "optimal" value by reducing the differences between doses measured / calculated. The objective of this work was to measure the FT and DLG of a TrueBeam accelerator (TB) with different setups and detectors, model these parameters and validate the results applying the AAPM-TG119 methodology using the Portal Dosimetry (PD) system. To obtain FT and DLG (6MV, 6FFF, 10MV and 10FFF), DICOM plans supplied by Varian and Farmer ionization chamber and CC13 were used in different setups. For the modeling, the dose measured with the Farmer chamber was initially used in VMAT plans for prostate and head and neck. From the obtained data, interactively the values of the FT and DLG were altered by searching for a higher oil index (> 95\%) in the $1 \% 1 \mathrm{~mm}$ criterion using the PD. Finally, all planning of TG119 with IMRT and VMAT were measured using $P D$. The measured values were: $6 X[(F T=1.1 \% / 1.0 \%)(D L G=0.044 \mathrm{~cm} / 0.12 \mathrm{~cm})], 10 X$ $[(F T=1.3 \% / 1.3 \%(F T=0.93 \% / 0.4 \%)(D L G=0.036 \mathrm{~cm} / 0.05 \mathrm{~cm})], 10 F F F[(F T=1.14 \% / 1.05 \%)(D L G=$ $0.048 \mathrm{Cm} / 0.05 \mathrm{~cm})]$. The mean acceptance percentages for the gamma criterion $1 \% 1 \mathrm{~mm}$ of the TG119 schedules with VMAT / IMRT after modeling were 6X: 96\% / 76\%; 10X: 98\% / 95\%; 6FFF: 97\% / 87\%; 10FFF: $97 \% / 82 \%$. The modeling of FT and DLG is useful to improve the accuracy of patient quality assurence, but the choice of detector and the technique used (VMAT or IMRT) are determinant and lead to different results. A balance should be found according to the routine of the department.
\end{abstract}

Keywords: radiotherapy; VMAT; transmission factor; Dosimetric Leaf Gap; modeling.

\section{Introdução}

O sistema de colimação MLC (do inglês, Multi Leaf Collimator), fundamental para as técnicas de radioterapia de intensidade modulada, IMRT
(Intensity Modulated Radiation Therapy) e VMAT (Volumetric Modulated Arc Therapy) (1), requer alta precisão tanto mecânica quanto dosimétrica de forma a garantir a qualidade do tratamento. 
Para MLC com a ponta arredondada, o sistema de planejamento Eclipse (Varian) exige a medida do FT (Fator de transmissão) e DLG (Dosimetric Leaf Gap) para cálculos de IMRT e VMAT. O FT considera a transmissão de radiação inter e intralâminas enquanto o DLG quantifica a transmissão nas extremidades das lâminas e, possibilita corrigir uma limitação do Eclipse que não considera o design arredondado do MLC em sua modelagem intrínseca. Estudos indicam que a utilização desses fatores obtidos, experimentalmente, pode não ser suficiente para uma boa concordância entre as doses planejadas e medidas durante os controles da qualidade individuais de pacientes, devido a incertezas inerentes ao procedimento experimental proposto para obtenção do FT e DLG (2-4). Diante disso, este trabalho tem por finalidade avaliar diferentes setups e detectores para determinação dos FT e DLG de um AL (Acelerador Linear) TrueBeam, realizar uma modelagem desses fatores para obter um valor "ótimo" capaz de aumentar a precisão da dose entregue em relação à calculada pelo sistema de planejamento durante os controles da qualidade de pacientes e, aplicar a metodologia da AAPM-TG119 para cada energia, nas técnicas de IMRT e VMAT após a modelagem.

\section{Materiais e Métodos}

A fim de se obter o FT e o DLG para cada energia de fótons do acelerador linear TrueBeam (HD120 "High Definition") (6MV, 10MV, 6FFF e 10FFF), utilizou-se planos DICOM fornecidos pelo próprio fabricante (Varian). Cada plano apresenta 10 campos de tamanho $10 \times 10 \mathrm{~cm}^{2}$ : campo 1 aberto; campos 2 e 3 com MLC estático de determinação de transmissão das lâminas dos carros $A$ e $B$, respectivamente (representados na figura 1); e, campos, de 4 a 10, com MLC dinâmico com gap medindo 2, 4, 6, 10, 14, 16 e $20 \mathrm{~mm}$. Cada campo foi irradiado com 100 UM, com duas câmaras de ionização diferentes (Farmer e CC13) e duas técnicas (Isocêntrica e SSD $=100 \mathrm{~cm}$, ambas com a câmara posicionada a uma profundidade de $5 \mathrm{~cm}$ na água), a fim de se avaliar a influência da técnica e do instrumento de medida sobre os valores obtidos. Um gráfico (Gap $(\mathrm{mm})$ vs Carga $(\mathrm{nC})$ ), representado na figura 2, é gerado a partir das seguintes equações:

$$
\begin{gathered}
R_{g T}=R_{T} \cdot\left(1-\frac{g[m m]}{120[\mathrm{~mm}]}\right) \\
R_{g}{ }^{\prime}=R_{g}-R_{g} T
\end{gathered}
$$

$\mathrm{Na}$ equação 1, $\mathrm{R}_{\mathrm{g} T}$ representa a carga correspondente à transmissão inter e intralâminas, $\mathrm{R}_{\mathrm{T}}$ é a média dos fatores de transmissão das lâminas dos carros A e B; e, g representa o tamanho do gap. $\mathrm{Na}$ equação $2, \mathrm{Rg}$ representa a leitura de carga total de cada campo com seus respectivos tamanhos de gap, enquanto que Rg' representa a carga correspondente ao gap, ou seja, descontando-se a carga medida devido a transmissão inter e intralâminas, calculada na equação 1. Por meio da equação de reta, é obtido o valor que intercepta o eixo y $(\operatorname{Gap}(m m))$, representando o valor de DLG (fator b na equação 3). Considerando as pontas arredondadas das lâminas, ainda que não houvesse gap, existe transmissão devido a esse design. Como o sistema de planejamento Eclipse não é capaz de considerá-lo, o valor de DLG encontrado representa o gap a ser considerado pelo TPS para que a leitura de cargas fosse a mesma que em situação de gap zero.

Os valores obtidos foram comparados entre si e com valores de referência (5) (tabela 1). Em seguida, foram inseridos no Eclipse os valores de FT e DLG obtidos sob a utilização da câmara Farmer a partir do setup isocêntrico. Planejamentos tratados de próstata e cabeça e pescoço, realizados na técnica VMAT para cada energia de fótons, foram calculados no TPS e medidos utilizando-se câmara de ionização. A diferença entre as doses entregues e calculadas foram avaliadas. Os valores de FT e DLG foram alterados no Eclipse e, os planejamentos de próstata e de cabeça e pescoço, calculados e medidos novamente. Uma modelagem foi realizada, repetindo-se esse procedimento. Posteriormente, os planejamentos foram medidos a partir do PD (detector de escolha do serviço) e, uma nova etapa do processo de modelagem foi realizada alterando-se os valores de FT e DLG até que uma maior concordância entre doses fosse alcançada, neste caso, buscando um maior índice de aceite (>95\%) no critério gamma de $1 \% 1 \mathrm{~mm}$ para planejamentos de VMAT.

Após a modelagem, já com os valores "ótimos" de FT e DLG, todos os planejamentos do TG119 foram realizados nas técnicas IMRT e VMAT, nas quatro energias de fótons. Os planejamentos foram executados e medidos por meio da utilização do PD.

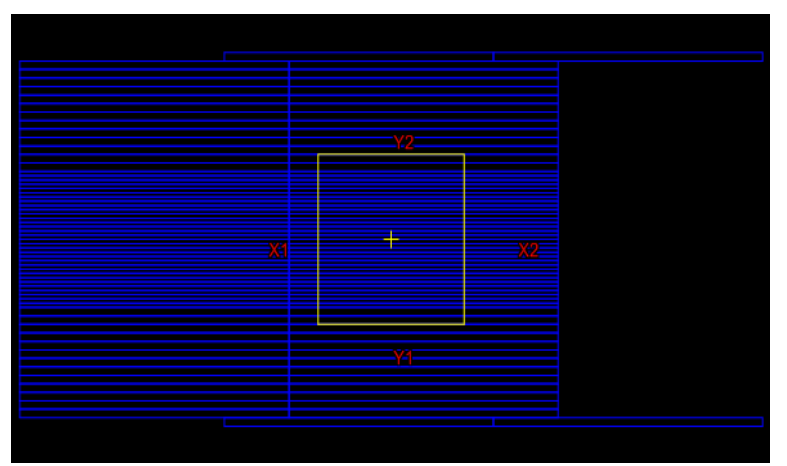

Figura 1. Campo com MLC estático de determinação de FT referente ao carro $A$. 


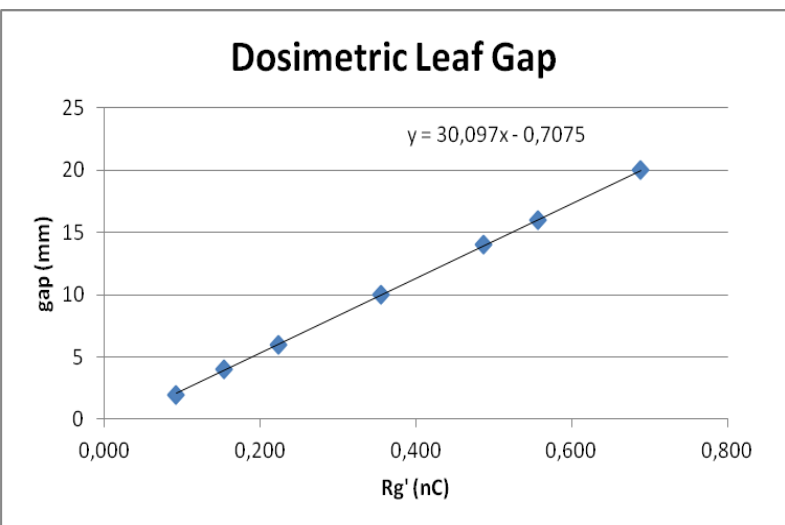

Figura 2. Representação do gráfico gerado a partir das leituras de carga obtidas para cada tamanho de gap para determinada energia.

\section{Resultados}

Os valores de FT e DLG obtidos para cada setup e câmara estão expressos na tabela 1 .

Os resultados mostram que, independente do instrumento de medida e da técnica, quanto maior a energia, maior o FT e, maior o DLG. Além disso, para as energias sem filtro aplanador (FFF), o FT e o DLG foram menores em relação às respectivas energias na presença do filtro.

Tabela 1. FT e DLG obtidas na técnica isocêntrica e SSD $=100 \mathrm{~cm}$, utilizando câmara Farmer e CC13

\begin{tabular}{|c|c|c|c|c|c|c|c|c|c|c|}
\hline \multirow[b]{3}{*}{ Energia } & \multicolumn{6}{|c|}{ FARMER } & \multicolumn{4}{|c|}{ CC13 } \\
\hline & \multicolumn{2}{|c|}{ Isocêntrico } & \multicolumn{2}{|c|}{ SSD 100} & \multicolumn{2}{|c|}{ Referência } & \multicolumn{2}{|c|}{ Isocêntrico } & \multicolumn{2}{|c|}{ SSD 100} \\
\hline & $\mathrm{FT}(\%)$ & DLG(cm) & FT(\%) & $\mathrm{DLG}(\mathrm{cm})$ & $\mathrm{FT}(\%)$ & DLG(cm) & $\mathrm{FT}(\%)$ & DLG(cm) & $\mathrm{FT}(\%)$ & DLG(cm) \\
\hline $6 X$ & 1,11 & 0,0439 & 1,12 & 0,0465 & 1,30 & 0,082 & 1,19 & 0,0582 & 1,23 & 0,0708 \\
\hline $10 \mathrm{X}$ & 1,30 & 0,0548 & 1,29 & 0,0548 & 1,60 & 0,093 & 1,36 & 0,075 & 1,41 & 0,0927 \\
\hline $6 \mathrm{FFF}$ & 0,93 & 0,0362 & 0,92 & 0,0342 & 1,10 & 0,071 & 1,05 & 0,0607 & 1,08 & 0,0659 \\
\hline $10 \mathrm{FFF}$ & 1,14 & 0,0477 & 1,13 & 0,0476 & 1,40 & 0,089 & 1,27 & 0,0617 & 1,27 & 0,0792 \\
\hline
\end{tabular}

Os valores de FT e DLG obtidos foram também menores do que os valores utilizados como referência, independente da comparação ser feita com o mesmo setup ou setups diferentes ao de referência.

Utilizando os valores de FT e DLG obtidos a partir da câmara Farmer e técnica isocêntrica, a comparação entre doses medida e calculada em câmara de ionização, apresentou um desvio médio percentual de $2,5 \%$ para a energia de $6 \mathrm{X}$ e de $3,5 \%$ para energia de $10 \mathrm{X}$. Após a modelagem de ambos os fatores, o desvio percentual de dose foi reduzido para $0,1 \%$ e $0,6 \%$ respectivamente.

A porcentagem de aceite da análise gama dos planos do TG119 realizados com o PD estão na tabela 2 com critério gama de $3 \% / 3 \mathrm{~mm}$ e, na tabela 3, com critério gama de $1 \% / 1 \mathrm{~mm}$.

Tabela 2. Porcentagem de aceite do critério gama $3 \% / 3 \mathrm{~mm}$ de cada energia, nas técnicas IMRT e VMAT.

\begin{tabular}{|c|c|c|c|c|c|c|}
\cline { 2 - 7 } \multicolumn{1}{c|}{} & \multicolumn{3}{c|}{ IMRT (\%) } & \multicolumn{3}{c|}{ VMAT (\%) } \\
\cline { 2 - 7 } & Média & Maior & Menor & Média & Maior & Menor \\
\hline $\mathbf{6 X}$ & 99,4 & 100 & 97,7 & 99,98 & 100 & 99,9 \\
\hline $\mathbf{1 0 X}$ & 99,5 & 100 & 97,7 & 100 & 100 & 100 \\
\hline $\mathbf{6 F F F}$ & 100 & 100 & 100 & 100 & 100 & 100 \\
\hline $\mathbf{1 0 F F F}$ & 98,9 & 100 & 95,2 & 100 & 100 & 100 \\
\hline
\end{tabular}

Tabela 3. Porcentagem de aceite do critério gama $1 \% / 1 \mathrm{~mm}$ de cada energia, nas técnicas IMRT e VMAT.

\begin{tabular}{|c|c|c|c|c|c|c|}
\cline { 2 - 7 } \multicolumn{1}{c|}{} & \multicolumn{3}{c|}{ IMRT (\%) } & \multicolumn{3}{c|}{ VMAT (\%) } \\
\cline { 2 - 7 } \multicolumn{1}{c|}{} & Média & Maior & Menor & Média & Maior & Menor \\
\hline $\mathbf{6 X}$ & 76 & 82,7 & 70,8 & 96 & 98,6 & 93,1 \\
\hline $\mathbf{1 0 X}$ & 87 & 99 & 76,3 & 97 & 99,2 & 94,2 \\
\hline $\mathbf{6 F F F}$ & 95 & 100 & 83,7 & 98 & 98,8 & 95,1 \\
\hline $\mathbf{1 0 F F F}$ & 82 & 87,6 & 74,3 & 97 & 98,8 & 94,4 \\
\hline
\end{tabular}

Os resultados das tabelas 2 e 3 mostram um maior nível de aceite na técnica VMAT em relação a técnica IMRT. Embora a técnica IMRT tenha apresentado uma porcentagem de aceite menor que para o VMAT no critério $1 \% / 1 \mathrm{~mm}$, todos os planejamentos no critério $3 \% / 3 \mathrm{~mm}$ ficaram com porcentagem de aceite acima de $95 \%$.

Os valores obtidos a partir da metodologia proposta pela Varian e os modelados usando o PD se encontram na tabela 4.

Tabela 4. FT e DLG medidos e FT e DLG obtidos após modelagem.

\begin{tabular}{|c|c|c|c|c|}
\cline { 2 - 5 } & \multicolumn{2}{c|}{ Medidos } & \multicolumn{2}{c|}{ Modelados } \\
\cline { 2 - 5 } & FT (\%) & DLG (cm) & FT (\%) & DLG (cm) \\
\hline 6X & 1,11 & 0,044 & 1 & 0,12 \\
\hline 10X & 1,3 & 0,055 & 1,3 & 0,13 \\
\hline 6FFF & 0,93 & 0,036 & 0,4 & 0,05 \\
\hline 10FFF & 1,14 & 0,048 & 1,05 & 0,05 \\
\hline
\end{tabular}

A partir da tabela 4, observa-se que os valores de FT após a modelagem foram menores ou iguais aos valores iniciais. Entretanto, os valores de DLG foram maiores, chegando a um percentual de $273 \%$ maior que o os obtidos experimentalmente. 


\section{Discussão}

Valores maiores de FT e DLG para as altas energias já eram esperados devido ao maior poder de penetração de feixes mais energéticos. Além disso, a ausência do filtro aplanador faz com que a energia média do feixe seja menor em relação ao feixe de mesma energia com a presença do filtro, ou seja, o feixe torna-se menos endurecido na ausência do filtro. Consequentemente, a carga coletada pela câmara de ionização é menor em feixes FFF, resultando em FT e DLG, também, menores.

Os resultados mostram que a dependência do DLG em relação ao setup e às câmaras é maior que do FT. Além disso, câmaras com volume sensível menor (CC13) são mais influenciadas pela mudança de setup.

Dessa forma, os valores de escolha a serem inseridos no Eclipse e modelados foram os obtidos a partir da câmara de ionização do tipo Farmer, devido a sua maior sensibilidade e estabilidade, no setup isocêntrico.

Embora o desvio percentual entre as doses tenha sido baixo após a modelagem feita a partir dos dados obtidos pela câmara de ionização, ao se medir os planos no PD, esses desvios se mostraram maiores. Assim, tornou-se necessária uma nova modelagem feita a partir do detector de escolha do serviço.

Por mais que a técnica escolhida como base para a modelagem tenha sido o VMAT, todos os planejamentos passaram no critério de aceite em ambas as técnicas utilizando-se o critério $3 \% / 3 \mathrm{~mm}$ (critério adotado no serviço para controles da qualidade de pacientes).

\section{Conclusões}

A diferença nos valores de DLG medidos e modelados mostra que sua determinação pelo método proposto não é suficiente. Isso sugere que, além das medidas e obtenção do DLG por meio de extrapolação gráfica, sejam feitas manipulações e testes experimentais utilizando o Eclipse para uma modelagem dos parâmetros. Apesar de os controles da qualidade terem melhorado após a modelagem do DLG a partir da utilização da câmara de ionização, uma nova modelagem se fez necessária a partir de planejamentos simulando outros tipos de tratamento e, a partir de outro detector.

Os resultados também mostraram que a modelagem com detectores diferentes chegam a resultados diferentes do índice gama, o que sugere que a modelagem deve ser feita com 0 detector que será utilizado na prática clínica.

Observando a porcentagem de aceite para as duas técnicas (IMRT e VMAT), pode-se concluir que a modelagem feita através de uma técnica de tratamento, pode não ser a ótima para outra. Caso o usuário deseje obter porcentagens de aceite maiores para as duas técnicas, é necessária a modelagem do FT e do DLG para ambas e criação de duas máquinas no sistema de planejamento Eclipse.

Desta forma, nota-se que a modelagem do FT e DLG é útil para melhorar a precisão dos controles da qualidade dos pacientes, porém, o detector de escolha e a técnica usada são determinantes e levam a diferentes resultados. Um equilíbrio deve ser encontrado de acordo com a rotina do departamento.

\section{Agradecimentos}

À equipe de física médica em radioterapia do Hospital de Câncer de Barretos.

\section{Referências}

1. Jeraj M, Robar V. Multileaf collimator in radiotherapy. Radiol Oncol. 2004;38(3):235-40.

2. Yao W, Farr JB. Determining the optimal dosimetric leaf gap setting for rounded leaf-end multileaf collimator systems by simple test fields. J Appl Clin Med Phys. 2015 Jul 08;16(4):5321.

3. Ajo Jr R. A dosimetric evaluation of the Eclipse and Pinnacle treatment planning systems in treatment of vertebral bodies using IMRT and VMAT with modeled and commissioned flattening filter free (FFF) fields. 2016.

4. $\quad$ Szpala S, Cao F, Kohli K. On using the dosimetric leaf gap to model the rounded leaf ends in VMAT/RapidArc plans. Journal of applied clinical medical physics. 2014;15(2):67-84.

5. $\quad$ Chang Z, Wu Q, Adamson J, Ren L, Bowsher J, Yan $\mathrm{H}$, et al. Commissioning and dosimetric characteristics of TrueBeam system: composite data of three TrueBeam machines. Med Phys. 2012;39(11):6981-7018. 